\title{
Towards a Definition of Digital Information Preservation Object
}

\author{
Hugo Quisbert, Margarita Korenkova, Ann Hägerfors
}

\begin{abstract}
In this paper, we discuss long-term digital preservation from an information perspective, rather than the predominant approaches; the Archival and the Technocratic Approach. Information lives longer than people, tools (software) and organizations live. The Information Continuum Model provides support for this standpoint. However, we find that there exists no concept to support practical action in preservation from the information perspective. Existing concepts as information object, digital object, preservation object, electronic record, information package and significant properties are context dependent and focus on the object to be preserved, rather than preservation of information. Consequently, they are not suitable for realizing the information perspective in long-term digital preservation. The concept of Digital Information Preservation Object is therefore introduced and a tentative definition of the concept is presented.
\end{abstract}

\section{Introduction}

During the last years, development of digital technologies has been very fast and for the most unpredictable. Consequently, the amount of information created in electronic form is growing exponentially. [18] According to Runardotter et al., [25] digital information has to be available for the future society by legal, historical and democratic reasons.

The solutions that are today for preservations and foremost to make digital material accessible for long time are insufficient. Archival inquiry "Archives for Everyone - Now and in the Future", called attention to these great shortcomings and argued that models, methods and platform-independent software for preservation of the digital material are omitted largely. [19] On one hand, the "problem" with digital preservation is the lack of proven methods to ensure that the digital information will continue to exist [26]. On the other hand, the software currently available does not include good tools for saving digital recorded information in the face of rapid hardware and software obsolescence [12]. One of the challenges to be addressed is how to preserve information through technology

Hugo Quisbert, Luleå University of Technology, Division of Systems Sciences

University Area, 97187 Luleå, Sweden, Hugo.Quisbert@1tu.se

Margarita Korenkova, Luleå University of Technology, Division of Systems Sciences

University Area, 97187 Luleå, Sweden, Margarita.Korenkova@1tu.se

Ann Hägerfors, Luleå University of Technology, Division of Systems Sciences

University Area, 97187 Luleå, Sweden, Ann.Hagerfors@1tu.se 
changes. [12] Within the practice of preservation, archives are still using methods developed since 1970-1980. These methods cause losing functionalities of records when archiving. Archival Science has not developed as rapid as information technology. Digital material is needed to be migrated and/or emulated to be preserved. [25]

Information Systems Sciences have no methods that in the same degree as Archival Science deal with long-term, "forever" perspectives. The transition between paper-based media to digital media was not preceded by a strategy for long-term digital preservation. This phenomenon explained in terms of lack of knowledge and experience when handling digital material. [25]

Society and information curators have to face the challenge of finding some sustainable solutions to ensure that digital recorded information of today become accessible and understandable in the future. Otherwise, digital material and likewise one part of society's future cultural heritage and history are in danger of disappearing forever. Consequently, legal and democratic demands cannot be fulfilled. [19] According to Gladney [12] technology has influence in almost every aspect of long-term digital preservation and it is not widely believed these solutions can be achieved solely through technological means. To find solutions to the problem much research was limited to technical aspects of preservation but our research has taken another view on long-term digital preservation, which puts information in focus. This paper therefore discusses the concept of information as central for long-term digital preservation.

\section{Facing Long-term Digital Preservation}

The authors of this paper are connected to the Long-term Digital Preservation Centre (LDP Centre) in Sweden. The major activities of the LDP Centre concern research and systems development within the field of long-term digital preservation. The LDP Centre has adopted, at this stage, migration as method for preservation and developed a model for structure for Information Package in accordance with [4]. This structure was (and is) intended to be used by archive creating organizations, in order to later deliver digitally recorded information to archives. The base-structure consists of different sets of metadata according to well-established international metadata standards such as METS, EAC, EAD, PREMIS, etc.

We found out that the existing models for structuring information about the object to be preserved, produces problems in implementation. As we can realize, these structures are used as "wrappers" carrying metadata sometimes overlapping one another. Consequently, dealing with all these structures causes both intellectual complications and technical implementation difficulties. We, at the LDP Centre, have experienced that all these structures have some kind of inherent philosophy, which focuses on special features, or attributes of digital material. Here we did three significant observations: 1) the used structures do not focus specifically on information, 2) using several structures sometimes causes an 
intellectual "mess" due to their inherent philosophies (such as ontological confusion), and 3) the used structures are inherited from the archival world and they support legislative (or authority) records more than digitally recorded information in general. We also found out that putting many efforts on these structures, made us loss focus on that we believe is important to preserve, information. It is obvious that technical or other aspects of digital material to be preserved are indeed important to deal with, but those are just means of preserving information while the digital "technology is a live".

Another issue concerns the fact that it was archival institutions that early experienced the need of digital preservation. Consequently, (archival) metadata standards were developed in order to support digital preservation. The archival inheritance spokes for the principle of provenience as one of the stone corners in long-term digital preservation, because this principle supports authenticity and trustworthiness of any kind of digitally recorded information. Consequently, today's "solutions", based on the Archival and/or the Technocratic Approach supports preservation only in a (relatively) short-term.

To approach a solution concerning how to preserve information through technology changes and surpassing the archival inheritance, the LDP Centre's research activities have taken a new view, which puts information in focus. This includes the development of a detached Digital Information Preservation Object that is independent of technology and deals with the question of provenience in a rather new way. Such preservation object might be transferred between different technologies. This is viewed as the main key to a viable solution for long-term digital preservation. Such view demands a higher-level perspective of information and its continuing value without neglecting the constant changing technical aspects of digital preservation.

\section{Developing the Information Approach}

This paper is a result of two research activities, literature review and interaction in the developing R\&D team at the LDP Centre. The literature review was carried out with the purpose of developing a definition of Digital Information Preservation Object and the interaction helped to gather data about the developed structure through seminars, (informal) "interviews" and systems development meetings.

There is a large quantity of literature about the concept of information. Nevertheless, in comparison, the quantity of literature in the field of long-term digital preservation is relatively small. The process of critical selection of relevant literature was based on guidelines such as "well known writers", "established perspectives and/or schools", in order to address the key sources, key theories and definitions of the topic [13]. Since our intention was to provide a broad view on the subject, the initial literature review made it clear that several research projects have shown its own approaches to what we call Digital Information Preservation Object. In our work, we tackle questions like: What are the elements of the 
Information Approach? What properties/characteristics constitute a definition of the notion Digital Information Preservation Object?

Since the LDP Centre intends to develop a general preservation model in which Digital Information Preservation Object is the mediator for preservation, this paper will have influence for further research as well as for what methods, models and techniques that will be developed and used. Furthermore, our result will influence the development of the information systems intended to serve long-term digital preservation.

\subsection{On Information}

The term information is used with different meanings by different groups and in different contexts [17] and there is no well-defined definition of the terms "data" and "information" [6]. A common and short definition is that information is interpreted data [3]. Hence, data is signs used to represent information [17], or signs which carries with them, the possibility to compose and/or decompose the text, photo, music etc. from which it is derived. Thus, retrieving the source provides access to information and we can start reading/viewing/listening but also interpreting and analysing the information existing in the source. This results in the creation of knowledge, that is, we learn from information.

The central idea is that data become information by a process of interpretation [17]. Langefors [ibid.] states, "One of the central insights from infology is that data or texts do not "contain" information (knowledge) but will only, at best, represent the information to those who have the required "pre-knowledge"". Dealing with information systems require a broad view of information, since using a computer implicitly involves information services of some kind, that is, we get service by being informed by data. According to Langefors [17] it is necessary to define information as knowledge, since "information is knowledge and not physical signs". He regards information as knowledge structured in such a way that it is communicated. Because of this, it can also be stored, which leads to information being stored knowledge. [17]

Checkland \& Holwell [6] provides the view that information is a service that supports decision making within organizations. Data are facts and a starting point for mental processes. They introduce the concept of "capta", which is the result of selection of certain data (we pay attention to, create some new category or get surprised by the data). In other words, data that catches our interest transforms to capta, the consciousness of something. This signifies that turning data into information is done through a mental process and during that process the data changes shape, and will ultimately lead to knowledge. Once we have selected, paid attention to or created some data, or turned it into capta, we relate it to other things or put it in a context, we attribute meaning to it, and we once again convert it, this time to information. This can be done individually or collectively and this process, selection and conversion of data into meaningful information can lead to larger structures of interconnected information, or what we call knowledge. The 
interpretation process [17] emphasizes is implicit in this reasoning. We agree with Langefors with his notion that in order to understand information people has to have pre-knowledge. This we see as knowledge that consists of the ability to assimilate the information, but also to have an understanding of the information content, or the subject in focus. If these abilities are found, new knowledge is created the moment someone (individually or in a group) thinks of an issue. When people's ideas, or knowledge, are being shaped and transferred to a medium they are made available for others to share. Hence, the knowledge has now once again become information and exists physically (or digitally), available for people who need it in order to learn and increase their knowledge of something, for example within an organization.

\subsection{Information Continuum Perspective}

Runardotter et al., [25] put information in a long-term digital preservation context that is a new circular view of the information life cycle. The authors manifested that information is a living concept and is in progress during constant ongoing process. The statement is based on that information is constantly shaped and reshaped, evolving through time, picking up further information or losing some parts and in that way information is all the time under reconstruction. To keep information alive demands a set of ongoing activities, both organizational and technical. The idea of living spiral is also supported by the Records Continuum Model and Information Continuum Model [32] [33]. According these models information and records continues to evolve in space/time and therefore are no end products. [25] The named models are founded on Giddens' structuration theory. This theory of time/space distanciation has been translated into a "rhythm" for information processing derived from the processes of creating information, capturing it as recorded information, organising it and bringing it together within the plural domains of competition. Each item of information once created, may or may not be recorded, stored and managed in ways that can benefit individuals, groups, communities, and can push that information out into the plural domains of the further reaches of space/time. The continua (the dimensions of the model) in the Information Continuum Model are collectively meant to focus analytical attention on the nature of recorded information as an allocative resource, something to be shared and to be used within our activities. The continua in the Records Continuum Model collectively are meant to focus analytical attention on the nature of recorded information as an authoritative resource, something to be relied on not because of its content but because of the way, it has been created and maintained with some continuing contact to the original specificities of its occurrence. [33]

According to Upward [31], information (and its reconstituted products) outwardly spiral from the original act of creation of a document. In the Information Continuum Model, information is derived from a principle source (for example, an information object such as an interview), and becomes transformed 
through the agency of people and technologies for different purposes. Information Objects are both embedded in the "context of the action in which they are part, and are recursively involved". [28]

We could use Giddens own "words" and transform these to our vocabulary of Information Continuum. Giddens "structure" equals to rules and resources organized as properties of systems. Systems equal to reproduced relations between actors or collectivities organized as social practices. Finally, structuration equals to the continuity or transformation of structures, and therefore the reproduction of systems [16] [11]. The dynamic of structuration is crucial in our view. "Social practices evolve over time and space and has to replicate even to stay the same" [24] (the essence of long-term digital preservation). The term of continuity, (i.e. continuum) promotes the reproduction of systems (i.e. memory traces, organized social practices) by means of digitally recorded information and its continuing value. Information is seen as an (allocative) resource, something to be shared and to be used within our activities [33] [25]. In the long-term gaining access to digitally recorded information (through long-term digital preservation activities) will tell us what sorts of things were out there in the world. We could summarize our view in simple words, as digital preservation will ensure, through the continuing value of information, that social systems will be reproduced as memory tracks of human activities.

\section{Predominant Approaches in Long-term Digital Preservation}

In our research, we could observe two predominant approaches trying to define the object of preservation, Archival (including Records Management) and a technical (or merely a technocratic) perspective.

\subsection{The Object of Preservation in the Archival Approach}

One definition of Preservation Object is given by Dollar [8] as "Preservation Objects are those to be archived. These objects are physically compounded by set of files in maybe different formats. The objects logically are compounded by data - the content for instance birth date of a person, and metadata - that should describe the context when the birth date of a particular person was used".

Preservation makes the record a vital component in the memory of organizations, individuals and in some cases of society [29]. A record is more than just information, it is supposed to be trustworthy i.e. being able to serve as evidence, and support accountability. The time for preservation could vary from days or months up to hundreds of years. [10][29] The International Standards Organization definition of records is: "Information created, received, and maintained as evidence and information by organizations or person, in pursuance 
of legal obligations or in transaction of business." [15] In the Guide for Managing Electronic Records of the International Council on Archives, from an archival perspective, a similar definition of record is given as "recorded information produced or received in the initiation, conduct or completion of an institutional or individual activity and that comprises content, context and structure sufficient to provide evidence of the activity." Records can occur in different forms and representations. They are usually represented as logically delimited information objects, for example, as distinct documents. [14] However, increasingly we find records in the form of distributed objects, such as relational databases and compound documents.

According to Thomassen [29] a record has four characteristics, which make the record unique in relation to other types of information: 1) Records serve as evidence over actions and transactions, 2) Records support accountability, 3) Records are related to processes, i.e. information that is generated by, and linked to some work processes, and 4) Records are going to be preserved, some even for eternity.

Cox [7] have stated that the evidential value of a record can only exist if the content, structure and context are preserved. The context is the link between different records that belongs together and to the process where the record was created. According to Dollar [8], the electronic record is more of a logical entity, where integral parts can be managed at different places within an information system, or even in different information systems.

The question "What is a record" has been one of the fundamental issues in debates and discussions in archiving and digital preservation over the past twenty years. Attempts to arrive at general definition of an electronic record were made in the last decade by several research projects and have by now being replaced by more constructive approaches that define the properties and functionalities of electronic records, which need to be preserved in a given context. An electronic record can be understood as a package or a set of technical properties and possibilities, and of administrative context where some of these technical possibilities were put to use. [26]

In contrast, one approach in Digital Libraries that might have a significant difference is proposed by Nichols [21]. In this approach, the concept of "artefact" is central and defined as "a physical object produced at sometime in the past, and attesting to a given set of practices, thinking, and ways of viewing the world, but whose importance will be defined by present and foreseeable future needs and use. The value of the artefact is strongly influenced, but not completely determined by, the document/object's features that are unique". [21] An artefact is then an object with a set of values such as Evidential, aesthetic, Associational, Market and Exhibition value. An artefact is of value to the extent that it testifies to the information being original, faithful, fixed, or stable. Although, this approach does not mention the term record but "artefact", we interpret this approach to be similar to the Archival Approach.

The definitions of records are to some extent usable in our perspective. We perceive the evidentiallity of records as the major focus of Archiving and Records Management and less focus is given to records as informational source. 
Thomassen [29] gives emphasis on the need of preservation of records in order to be reused as memory traces. This encompasses our Continuum perspective, which is beyond of just records. The "big difference" is that our perspective is turning the precedence of information in relation to Archiving. Another difference is that our perspective deal with any kind of digitally recorded information as evidence of human activities not just with records as legal evidence.

\subsection{The Object of Preservation in the Technocratic Approach}

A definition of Digital Object is given by Blanchi \& Petrone [2] as "a unique identified data abstraction that encapsulates content and access policies while providing a high-level, self-describing, type definition. Digital Objects are described through the use of an abstract typing mechanism that we refer to as content type". The concept of Information Package of the OAIS Reference Model [4] states that data is interpreted using its Representation Information (described in metadata), which produces information, necessary to interpret that content. In order to preserve successfully such a generic Information Object, it is critical for an Open Archival Information System (OAIS) to identify clearly and understand the Data Object and its associated Representation Information, which together forms what, is called an Information Package. Objects, according to PREMIS [23], can be bitstreams, files or representations. Further, the notion Preservation Object can be compared to the approach of Persistent Object articulated by the US National Archives (NARA) and the San Diego Supercomputing Centre (SDSC). This method presumes that all records can be represented as objects with their specific characteristics and behaviour [26].

The concept of Significant Properties, proposed by the National Library of Australia (NLA) [20], are functions or characteristics of an object that are essential to the meaning of it. Preservation of digital objects, in this approach, are going to necessitate changes in look, feel, functionality or content if the object is to remain usable at all. In the Cedars project [5], Significant Properties were associated with the Underlying Abstract Form (UAF) of an object. Therefore, objects with the same UAF have the same significant properties. The PREMIS [23] working group, following on the work of OAIS, Cedars and NLA, defined Significant Properties as "characteristics of a particular object subjectively determined to be important to maintain through preservation actions", noting that "Significant properties may be objective technical characteristics subjectively considered to be particularly important, or subjectively determined characteristics". Further, the FEDORA architecture [22] conceptualize a Digital Object as having: 1) a structural kernel, which encapsulates content as opaque byte stream packages and, 2) an interface, or behaviour, layer that gives contextual meaning to the data in the Digital Object. One useful metaphor for a Digital Object is that of a cell. 
A similar approach is given by Saidis et al. [27] as Digital Object Prototypes (DOPs) that has constituent components as files, metadata, behaviours and relationships. A DOP is a digital object type definition that provides a detailed specification of its constituent parts and behaviours. Digital objects are conceived as instances of their respective prototypes. The approach to Digital Libraries proposed by Arms et al. [1] described the "digital object" in a slightly different manner. The main building blocks in this approach are: "digital objects", which are used to manage digital material in a networked environment. From a computing view, the digital library is built up from simple components, notably digital objects. A digital object is a way of structuring information in digital form. [1]

Very common are the terms: "data abstraction", "data" "bitstreams", "files", "instantiation", "characteristics and behaviour", "functions", "functionality", "objective technical characteristics", "structural kernel", "encapsulates opaque byte stream packages", "interface", "metadata", "behaviours and relationships", "manage digital material in a networked environment", "stored". The ontology of this approach shows that the terms utilized come from computer science in general and at some extent from Object Oriented terminology. This spokes for data management through information systems making data central. The term object is also important, being conceptualization of data (such as files, bitstreams, etc.) having some behaviour (its functionality or implemented functions in as information system). No emphasis is made on information neither on its contents as traces of human activities.

\section{Towards a Definition of Digital Information Preservation Object}

Every other approach to define the object of preservation has focused from different perspectives of preservation and from different characteristics of the digital object using its own ontology. Furthermore, we found that 1) there is no existing or established definition of Digital Information Preservation Object, 2) existing concepts are context dependent and focuses specifically on object to be preserved. After our study we came to conclusions that research carried out so far do not handle Digital Information Preservation Object from Information Continuum perspective and do not contribute with any definition of the notion Digital Information Preservation Object. However, the studied research results merely focus on the structure and physical nature of the digital object. Their described abstractions are conceptualizations of the underlying physical level. Nevertheless, although these approaches support our activities to some extent, the definitions provided by these approaches are not suitable for our view, hence; we experienced the emergence of an own definition of Digital Information Preservation Object that specifically focuses on information. However, this study gave us insights to take another perspective. To define the notion of Digital 
Information Preservation Object it is needed to take a standpoint. We cannot begin the definition from structure of the object; nonetheless, we will begin definition from a global view. If we start from the structure, we are viewing an object as a delimited entity at structural level, since our focus is on information, not the object itself. We want to distinguish the conventional view to the object that is a construction, a container or a carrier to hold together the elements (depending on level of conceptualization) from the view that is to see an object from continuum perspective. An object there is a piece or pieces of information that reflects human action in a social context in the continuum.

Our intention (or level of abstraction) lay merely in the general level or, so to say, we try to define DIPO at an "ethereal" level; we want to define the "thing" DIPO. We take stand in the philosophy of Dooyeweerd who stated that Meaning rather than Existence is the primary property in created reality [9]. In our perspective, this view encompasses the Information Continuum Model perspective in which put the continuing value of information (i.e. its reconstruction of human social actions) as the meaning (raison d'être) of the DIPO.

The DIPO is a recorded result of action of humans in the form of information pieces. This information is categorized in an information type from social context. These information pieces can have relationships to other information pieces either within its own physical carrier or toward other objects. The vital characteristic of the information object from continuum perspective is that new object can be created through the combination of information pieces that can be a piece of other objects. In that way, the information will be reused in another context and reproducing human social actions. It means that information can be related to processes (that is generated by, and linked to work processes). Furthermore, the information in the object has to serve as evidence over actions and transactions as well.

\section{References}

1. Arms W., Blanchi C., Overly E.: An Architecture for Information in Digital Libraries. D-Lib Magazine (1997)

2. Blanchi, C., Petrone, J.: An Architecture for Digital Object Typing. Corporation for National Research Initiatives. Reston, Virginia (2001)

3. Bratteteig, T and Verne, G.: Feminist, or merely critical? In search of Gender Perspectives in Informatics. In Mörtberg, C, ed. Where do we go from here? Feminist Challenges of Information Technology. Division gender and Technology. Luleå University of Technology, Luleå. pp. 39-56 (2000)

4. CCSDS: Reference Model for an Open Archival Information System (OAIS) - Blue book. National Aeronautics and Space Administration (2002)

5. Cedars Project Team and UKOLN: Metadata for Digital Preservation: the Cedars Project outline specification: draft for public consultation, unpublished, www.leeds.ac.uk/cedars.pdf (2000)

6. Checkland, P and Holwell, S.: Information, Systems and Information Systems making sense of the field. Wiley \& Sons Ltd, Chichester, England (1998) 
7. Cox, R. J.: Managing Records as Evidence and Information. Quorum Books.(2001)

8. Dollar, C M.: Authentic Electronic Records: Strategies for long-term access. Cohasset Associates Inc. (2000)

9. Dooyeweerd H.: A New Critique of Theoretical Thought . Vol. 1. The Edwin Mellen Press (1997)

10. Duranti, L.: Concepts, Principles, and Methods for the Management of Electronic Records. The Information Society (2001) 17, 271-279

11. Giddens A.: The Constitution of Society. Polity Press. (1984)

12. Gladney, H. M.: Preserving Digital Information. Springer (2007)

13. Hart, C.: Doing a Literature Review. Sage Publications Ltd (1998)

14. ICA Committee on Current Records in an Electronic Environment: Electronic Records: A Workbook for Archivists. Draft (2005)

15. International Standards Organization. Iso 15489-1.: Information and Documentation and Records Management part 1: General. International Standards Organization (2001)

16. Jones M., Orlikowski W., Munir K.: Structuration Theory and Information Systems: A Critical Reappraisal. In Mingers J. \& Willcocks L. (Eds.): Social Theory and Philosophy for Information Systems. Wiley (2004)

17. Langefors B.: Essays on Infology. Dept. of Information Systems. Göteborg University, Göteborg (1993)

18. Lim Siew Lin, Chennupati K. Ramaiah Pitt Kuan Wal: Problems in the preservation of electronic records. Library Review, (2003) Vol. 52, nr. 3, pp. 117-125

19. Marklund, K.: Archives for Everyone - Now and in the Future. Government Offices of Sweden. SOU 2002:78 (in Swedish) (2002)

20. National Libraries of Australia. Digital Preservation Policy (2002)

21. Nichols, S.: Artifacts in Digital Collections. 67th IFLA Council and General Conference. August 16-25 (2001)

22. Payette, S. and Lagoze, C.: Flexible and Extensible Digital Object and Repository Architecture (FEDORA). Department of Computer Science Cornell University. Springer-Verlag (1998)

23. PREMIS Working Group. Data Dictionary for Preservation Metadata: final report of the Premis Working Group. Dublin, OCLC Online Computer Library Center and Mountain View, RLG, www.oclc.org/research/projects/pmwg/premis-final.pdf (2005)

24. Rose J.: Structuration Theory and Information system Development - Frameworks for Practice. Proceedings of The $9^{\text {th }}$ European Conference on Information Systems. (2001)

25. Runardotter, M., Quisbert, H., Nilsson J., Hägerfors, A., Miriamdotter, A.: The Information Life Cycle - Issues in Long-term Digital Preservation. Proceedings IRIS 28 - Information system research seminar in Scandinavia (2005)

26. Ruusalepp, R.: Digital preservation in archives: An overview of Current Research and Practices. Swedish National Archives, Sweden (2005)

27. Saidis, K., Pyrounakis, G., Nikolaidou, M., and Delis, A.: Digital Object Prototypes: An Effective Realization of Digital Object Types. Department of Informatics and Telecommunications, Libraries Computer Center, University of Athens. SpringerVerlag (2006)

28. Stillman L. J. H. Understandings of Technology in community-Based Organizations: A Structurational Analysis. Dr. Thesis. Faculty of Information Technology, Monash University (2006)

29. Thomassen, T.: A first introduction to archival science. Archival Science, (2001) Vol. 1, pp 373-385

30. Upward, F.: Structuring the Records Continuum - Part One: Postcustodial principles and properties. First published: Archives and Manuscripts, (1996) 24 (2) 
31. Upward, F.: Structuring the Records Continuum, Part Two: Structuration Theory and Recordkeeping. First published: Archives and Manuscripts, (1997) 25 (1)

32. Upward, F.: Modeling the Continuum as Paradigm Shift in Recordkeeping and Archiving Process and Beyond - a Personal Reflection. Records Management Journal. (2000) Vol. 10, nr 3. pp 115-139

33. Upward, F.: Community Informatics and the Information Processing Continuum. Proceedings of the Constructing and sharing memory: community informatics, identity and empowerment conference. Centre for Community Networking Research (CCRN) (2006) 